\title{
The Evolution of Methicillin-resistant Staphylococcus aureus
}

\author{
Jaiden Tu1, Patricia M. Gray ${ }^{1}$ \\ 1 University of Western Ontario, Department of Biology
}

ABSTRACT: Since 1961, methicillin-resistant Staphylococcus aureus (MRSA) has evolved through both single locus gene variation and horizontal gene transfer. By the late 1970s, the emergence of new SCCmec allotypes marked the beginning of a worldwide MRSA pandemic. The continuous and rapid evolution of MRSA, in response to new antibiotics, remains a major public health issue worldwide.

KEYWORDS: Methicillin-resistant Staphylococcus aureus, Evolution, Horizontal Gene Transfer, MRSA, HGT, Superbug, SCCmec

This essay is part of a continuing series of student class work completed in a second-year biology course at The University of Western Ontario, introduced here. The essay illustrates one or more principles of evolution on a topic of the student's own choice.

Methicillin-resistant Staphylococcus aureus (MRSA) is a type of staph bacteria, commonly referred to as a superbug, and it is resistant to many antibiotics. There are two general types of MRSA: health care-associated MRSA (HA-MRSA) and community-associated MRSA (CA-MRSA), which have different genetic characteristics and were traditionally found in different locations ${ }^{1,2,3}$. MRSA can lead to more invasive and potentially fatal infections such as pneumonia and is the most common cause of skin and soft tissue infections in emergency rooms in the United States ${ }^{1}$.

As Staph bacteria, MRSA are commonly located on the skin or in the nose ${ }^{4}$. Approximately one-third of the Canadian population is carrying Staph bacteria at any given time, but not all these bacteria will be MRSA ${ }^{4}$. In addition, being a carrier of MRSA does not guarantee that an individual will become infected ${ }^{4}$. Non-infected carriers are referred to as being colonized ${ }^{1}$. MRSA can be spread by physical contact with a carrier or infected individual, by contact with infected bodily fluids, or in some cases, it may even be airborne ${ }^{4}$. The ancestors of MRSA are penicillin-resistant strains of $S$. aureus, also known as methicillin-susceptible $S$. aureus (MSSA), that arose in the 1940s and are still present today ${ }^{1,2,5}$. These penicillin-resistant strains utilize a plasmid-encoded penicillinase that hydrolyzes the $\beta$ lactam ring of penicillin, disrupting antimicrobial activity ${ }^{1}$. The introduction of methicillin provided the selective pressure required for the emergence of
MRSA in 19611,6. The methicillin resistant gene, mecA, was most likely acquired from a distantly related species through horizontal gene transfer (Figure 1)6. The methicillin-resistant penicillinbinding protein (PBP2a or PBP2'), encoded by mecA, does not exist in MSSA ${ }^{6}$. Methicillin resistance applies to the entire $\beta$-lactam class of antibiotics, including penicillin, cephalosporin, and carbapenems ${ }^{7}$. Thus, MRSA exhibits a very broad spectrum of resistance compared with penicillinresistant strains of $S$. aureus.

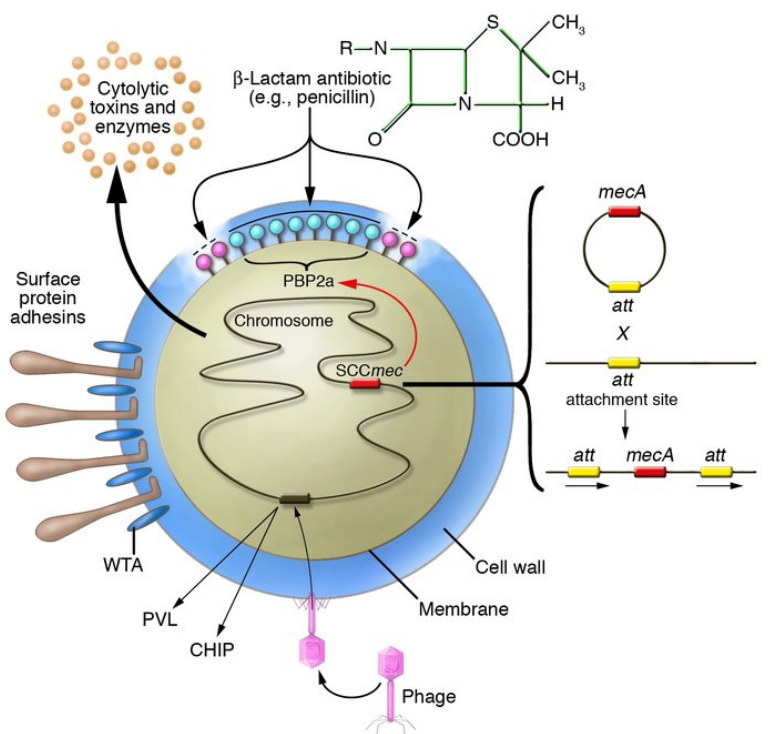


Figure 1 - Resistance to methicillin in Staphylococcus aureas. Surface protein adhesins and wall teichoic acid are expressed and are important factors in nasal and skin colonization. Resistance to methicillin is acquired by insertion of a horizontally transferred DNA element called SCCmec. Five different SCCmec elements can integrate at the same location by site-specific recombination. A novel $\beta$-lactam-insensitive penicillin binding protein, PBP2a, is encoded by the mecA gene?

There are two mechanisms by which MRSA can evolve: single locus variations from ancestral MRSA clones or horizontal gene transfer of mec determinants into MSSA ${ }^{5}$. MRSA evolution relies primarily on the method of gene transfer into MSSA lineages through the action of mobile elements, including SCCmec, vSa,$\phi S A 3$ and MES-related elements ${ }^{2}$.

MRSA isolates with the same sequence type (ST), but different staphylococcal cassette chromosome mec (SCCmec) types, suggest that the emergence of MRSA clones on multiple occasions is due to the transfer of mecA between $S$. aureus lineages ${ }^{5}$. The original MRSA clone is thought to be ST250-MRSA 5 . ST8, a successful MSSA strain that developed into ST250-MSSA via a single nucleotide polymorphism, acquired SCCmec type I, which becomes ST250-MRSA (Fig. 2)5.
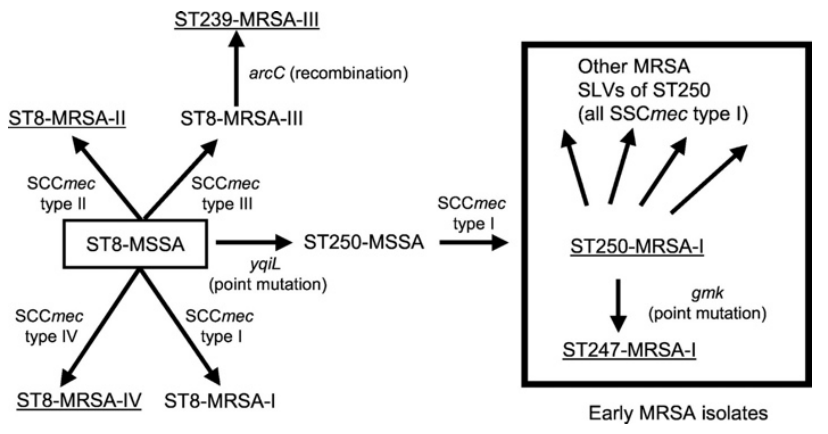

Figure 2 - Evolutionary origin of MRSA5.

Before the late 1970s, there was only one SCCmec allotype: SCCmecl1,7. The emergence of new SCCmec allotypes, SCCmecll and SCCmeclll, in MRSA marked the beginning of the worldwide pandemic that continues today ${ }^{1}$. The increased use of vancomycin -the last remaining antibiotic to which MRSA was readily susceptible - to treat MRSA led to vancomycin-intermediate $S$. aureus strains, which progressed to vancomycin-resistant $S$. aureus strains in the early 2000s ${ }^{1}$. Vancomycin resistance was likely acquired from Enterococcus, a vancomycin-resistant bacterium ${ }^{7}$. The emergence of community-associated MRSA (CA-MRSA) in the 1990s was a significant evolutionary event ${ }^{1}$. Although most antibiotics other than $\beta$-lactams are effective against CA-MRSA, it is an especially virulent strain of MRSA and is more easily transmitted ${ }^{1}$. The presence of two molecular markers not found in common hospital strains-a new $\mathrm{SCC} m e c$ allotype, SCCmedV, and various virulent factors including Panton-Valentine leukocidin (PVL) - indicates that CA-MRSA is unrelated to HAMRSA in terms of virulence and antibiotic resistance1. SCCmeclV, believed to have evolved from SCCmecl, is now the most widely distributed SCCmec type and is related to faster MRSA growth rates ${ }^{1}$.

A recent study of MRSA evolution in Taiwan led to the analysis of a new SCCmec type: SCCmecV2. The major Taiwanese ST59 MRSA clones-PVL-negative SCCmecIV and PVL-positive $\mathrm{SCCmecV}$-descended from a common ancestral MSSA genotype, CC59, whose vSa $\beta$ mobile element contained a $\phi S A 3-$ related fragment with immune evasion cluster (IEC) type $\mathrm{C}^{2}$. The specialization of PVL-positive SCCmecV began with the acquisition of MES $_{\mathrm{PM} 1}$ and the PVL-positive $\phi S A 2$ mobile element in CC59 MSSA, followed by the introduction of SCCmecV, converting MSSA to MRSA2. The translocation of mobile element $\phi S A 3$ and the gain

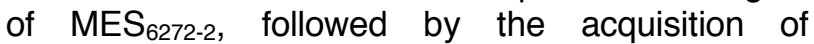
SCCmecIV, produced PVL-negative SCCmeclV MRSA $^{2}$. The success of ST59-MRSA clones results from increased antimicrobial resistance and virulence due to mobile genetic elements².

Another factor that contributes to MRSA reproductive success is bacterial colonization 2,7 . Beta-hemolysin causes the breakdown of red blood cells and influences the initial stages of MRSA nasal colonization ${ }^{2}$. Beta-hemolysin production is regulated by the $h / b$ gene $^{2}$. A novel mutation in CC59 $S$. aureus results in the ability to keep the $h b /$ gene active after acquiring IEC in the $\mathrm{VSa} \beta$ mobile element ${ }^{2}$. In addition, the removal of $\phi S A 3$ in the CAMRSA clone MW2 increases its ability to colonize skin2. Both the presence of IEC and active $h / b$ enhances MRSA's ability to colonize humans, leading to a rise in infection rates ${ }^{2}$. Acquiring these mutations that increase colonization is considered a selective advantage due to elevated opportunity for reproductive success. 
MRSA and MSSA continue to evolve as new antibiotics introduce novel selective pressures. The transfer of genes by mobile elements has a significant influence on the evolution of MRSA, increasing antibiotic resistance, virulence and colonization $1,2,7$. The continuous and rapid evolution of MRSA remains a major public health issue worldwide.

\section{References}

1. Chambers HF, DeLeo FR. Waves of resistance: Staphylococcus aureus in the antibiotic era. Nat Rev Microbiol. 2009;7(9): 629-641. DOI: 10.1038/nrmicro2200

2. Hung WC, Wan TW, Kuo YC, Yamamoto T, Tsai JC, Lin YT, et al. Molecular evolutionary pathways toward two successful community-associated but multidrug-resistant ST59 methicillin-resistant Staphylococcus aureus lineages in Taiwan: dynamic modes of mobile genetic element salvages. PLoS ONE. 2016;11(9): e0162526. DOI: 10.1371/journal.pone.0162526

3. Toronto Invasive Bacteria Diseases Network. Methicillinresistant Staphylococcus aureus: an update. TIBDN. 2012;4(3): 1-3. Available from: https://eportal.mountsinai.ca/Microbiology/research/tibdn/tib dnnews/TIBDN_Newsletter_16.pdf

4. Public Health Agency of Canada. Fact sheet - methicillinresistant Staphylococcus aureus [document on the Internet]. Toronto: PHAC; 2008 [cited 2017 Jan 5]. Available from: http://www.phac-aspc.gc.ca/id-mi/mrsa-eng.php

5. Enright MC, Robinson DA, Randle G, Feil EJ, Grundmann H, Spratt BG. The evolutionary history of methicillin-resistant Staphylococcus aureus (MRSA). Proc Natl Acad Sci USA. 2002;99(11): 7687-7692. DOI: 10.1073/pnas.122108599

6. Hiramatsu K, Cui L, Kuroda M, Ito T. The emergence and evolution of methicillin-resistant Staphylococcus aureus. Trends Microbiol. 2001;9(10): 486-493. DOI: 10.1016/S0966-842X(01)02175-8

7. Foster TJ. The Staphylococcus aureus "superbug." J Clin Invest. 2004;114(12): 1693-1696. DOI: 10.1172/JCI23825 